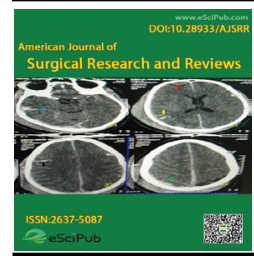

American Journal of Surgical Research and Reviews (ISSN:2637-5087)

\title{
Reconstruction of anterior frontal wall and contours defects using bone substitute
}

\section{Luigi Clauser, MD,DMD ${ }^{1}$, Antonio Lucchi, MD,DMD, Riccardo Luoni Orsi, DMD ${ }^{1}$ and Carolina Sannino MD ${ }^{1}$}

${ }^{1}$ Unit of Maxillo-Facial Surgery, Istituto Stomatologico Italiano, Via Pace, 21, 20122 MILANO, Italy

\begin{abstract}
Aim: The aim of this article is to report on the safety and long- ${ }^{*}$ Correspondence to Author: term efficacy of Cerament ${ }^{\circledR}$ BoneVoid Filler bone substitute for Prof. Luigi Clauser Unit of Maxilrepairing craniofacial bone defects. Post-traumatic cranioplasty is lo-Facial Surgery, Istituto Stomatoa complex and challenging procedure for all maxillo-craniofacial logico Italiano, Via Pace, 2120122 surgeons and neurosurgeons, especially when repairing large Milano, Italy, $\mathrm{Ph}+3902541761$ areas. The standard criterion for repairing small cranial defects is the use autogenous bone from the iliac crest or split calvarial grafts. Autogenous grafts may result in donor-site morbidity, increased surgical time, reabsorption, blood loss, and longer recovery time. Alloplastic materials used for bone repair, such as methyl methacrylate, hydroxyapatite, titanium, or porous polyethylene, are expected to have optimal properties, including easy adaptation, biocompatibility, ingrowth of new tissue, stability of shape, and low rate of reabsorption. A cranial implant should be easily shaped and positioned, allowing easy tissue growth. In very wide cranium defects the new technology is a custom made cranial implant constructed three-dimensionally with different types of materials. However, this procedure is very expensive with various infection rates depending on the kind of material used and on the chemicophysical composition of the implant.

Methods: The authors report the case of a 50-year-old man with a severe deformity of the forehead-supra orbital area as a result of a previous complex fronto-facial trauma treated in an emergency Unit. Secondary correction and reconstruction of the residual deformities were performed by using Cerament ${ }^{\circledR}$ Bone Void Filler, an alloplastic biphasic material, composed of $40 \%$

How to cite this article:

Luigi Clauser, Antonio Lucchi, Riccardo Luoni Orsi, Carolina Sannino. Reconstruction of anterior frontal wall and contours defects using bone substitute.American Journal of Surgical Research and Reviews, 2021, 4:34.

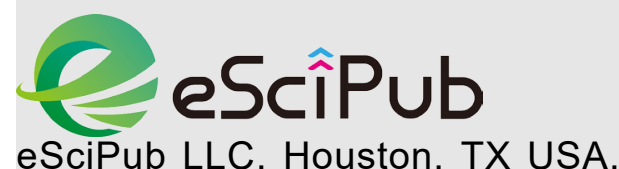
eSciPub LLC, Houston, TX USA. Website: https://escipub.com/ By using the site/services, you are agreeing to our Policies: https:// escipub.com/terms-privacy-policydisclaimer/
\end{abstract}


hydroxyapatite, $60 \%$ calcium sulfate and the radio-contrast agent iohexol. The unique ratio of hydroxyapatite and calcium sulfate is designed to enable Cerament to resorb at the same rate that bone forms. Calcium sulfate acts as a resorbable carrier for hydroxyapatite which is highly osteoconductive, promoting bone ingrowth.It seems to be a promising bone graft substitute in the management of bony irregularities in the fronto-orbital area.

Conclusion: The patient was first hospitalized as the result of a serious craniofacial trauma. One year after the first emergency cranio-orbital reconstructive operation, a marked deformity of the frontal region appeared with a "grid effect" due to the inadequate plate-bony fixation of the fractures applied during the first bony recomposition and because it was not as rigid as it should have been. A secondary surgery for deformity correction was performed. The hardware was totally removed and the bony deformity smoothed, reshaped, covered and filled using Cerament ${ }^{\circledR}$ Bone Void Filler, a biomaterial. The patient recovered with a satisfactory cranium-forehead shape, no complications, and complete disappearance of a frowning look of the fronto-orbital region.

Recently, increased use of bone substitutes in the reconstruction of bone defects has been fuelled by donor site complications associated with autologous bone harvesting. Cerament ${ }^{\circledR}$ BoneVoid Filler is a biphasic and injectable bone substitute that has a highly compressive strength and the ability to promote cancellous bone healing.

Keywords: Traumatology, fronto-orbital deformity, biomaterials, bone cement, bone grafting, cranioplasty, craniofacial trauma, craniofacial surgery, $f$ rontal sinus, trauma complications

\section{INTRODUCTION}

Frontal bone contour defects cause marked facial deformity with a visible morphologic and aesthetic impact. The etiology is usually posttraumatic or after craniofacial surgery for different reasons: previous surgical sequelae, congenital or acquired deformities, and post tumor with an intracranial approach.

There is still no ideal implant material for reconstruction of the forehead and relative supra-orbital area and contours. Even autogenous bone grafts have some drawbacks: source of bone grafts (cranium, iliac crest), donor site morbidity, longer surgical time, blood loss, volume maintenance over time and in some cases a limited bony harvesting area.

Cranial vault defects and deformities may also be repaired with alloplastic materials. ${ }^{[1]}$ Various materials can be used in place of autologous bone to reconstruct facial contours. There are some specific properties of these materials that need to be considered to reconstruct the frontal area. ${ }^{[2,3,4]}$

Ideally, alloplastic materials should have at least the following properties and qualities: easy adaptation, biocompatibility (which permits ingrowth of new tissue), stability of shape, reduced surgical times, and low rates of infection and reabsorption.

Biomaterial implants need to be easily shaped and positioned, allowing easy tissue ingrowth, and be able to stay in a secure position in addition to having satisfactory aesthetic outcomes in the craniofacial area. ${ }^{[5,6]}$

One of these materials is Cerament ${ }^{\circledR}$ Bone Void Filler (CBVF), a paste that has been successfully used to contour various bony and skull defects including reconstruction of the fronto-facial skeleton. It can be easily contoured to fill and cover a defect. ${ }^{[7,8]}$ It dries isothermally within several minutes. The substance then hardens to cement within four to six hours. It has been shown that, over time, the hydroxyapatite cement becomes replaced by bone without volume loss 
Luigi Clauser et al., AJSRR, 2021, 4:34

We observed that CBVF is gradually absorbed during postoperative weeks and months, leaving a natural and controlled internal bone growth, whereas hydroxyapatite acts like a long-term osteoconductive matrix into the surrounding bone

The focus of the present paper is on the safety and long-term efficacy of this bone substitute for repairing craniofacial bone defects, especially for restoration of frontal and upper-orbital bones and contours.

Safety is measured dually by resistance of the bone substitute to infection and foreign body reaction. The efficacy is evaluated as structural skull integrity, volume stability with time, aesthetic quality, and most importantly the capacity the bone substitute to adequately integrate into a cranium without subsequent deformities or complications. ${ }^{[9]}$


FIGURE 1 - A,B,C,D,E,F,G; Different views of fronto-supraorbital deformities after complex craniofacial trauma $(A, B, C, D, E)$. 3D CT scan shows the entire forehead deformity with bony fragment malposition, inappropriate bony rigid fixation and central forehead sinking $(F, G)$.

MATERIALS AND METHODS - CLINICAL of a severe fronto-naso-orbito-ethmoidal REPORT

A 50-year-old male was hospitalized with a diagnosis of acute craniofacial trauma as a result fracture, including the frontal and posterior walls of the frontal sinuses, and involving the roof and medial walls of both orbits. One year after the 
first emergent cranio-orbital reconstructive bony recomposition and ill-suited repositioning operation, a marked deformity of the frontal of re-osteotomized frontal bony fragments region appeared with a "grid effect." It was due (FIGURE 1 A,B,C,D,E,F,G,H).

to inappropriate rigid fixation of fractures during
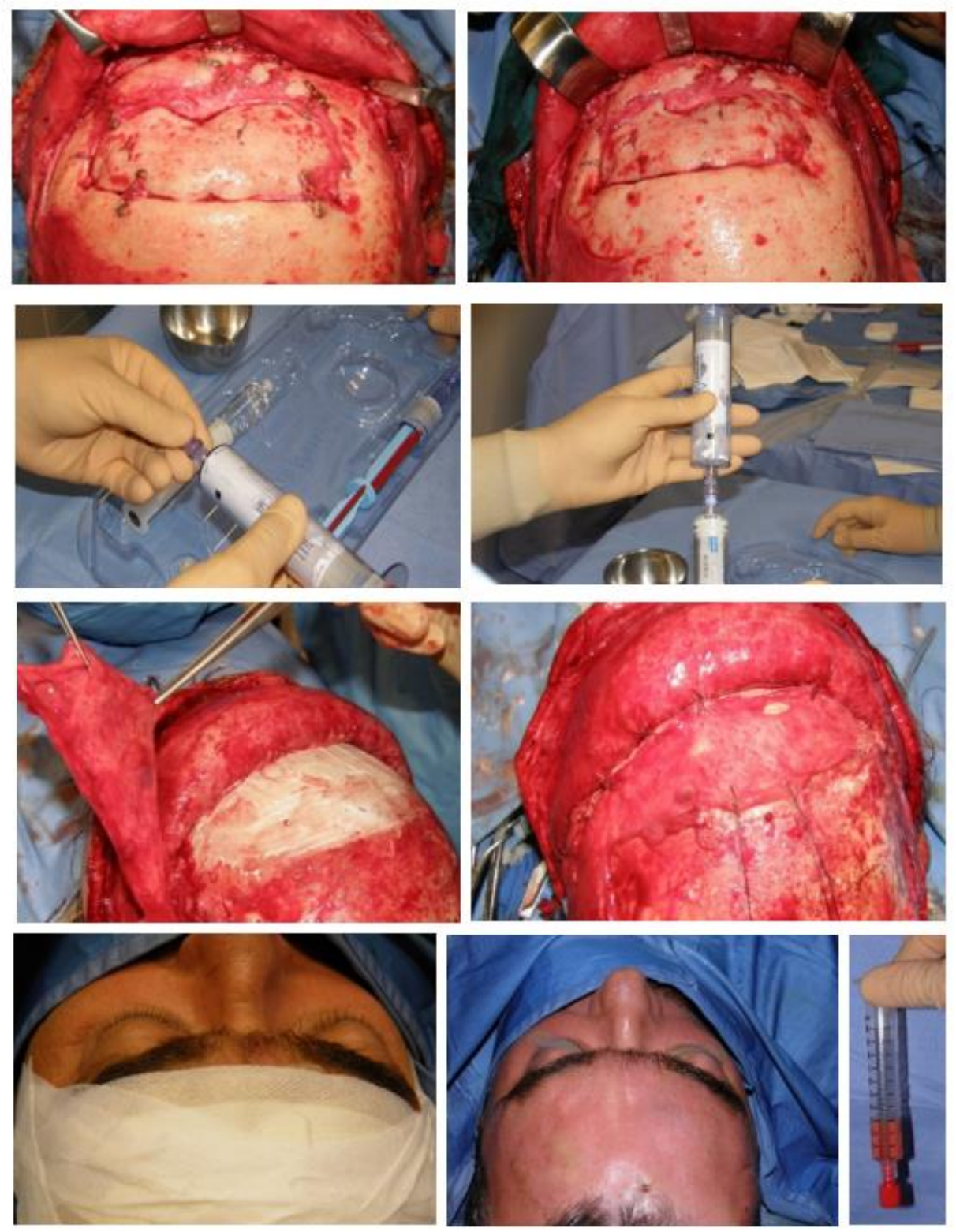

FIGURE 2 - A,B,C,D,E,F,G,H; Intraoperative views: bony malconsolidation before and after the hardware removal $(A, B)$ Preparation of the cement bony substitute Cerament Bone Void Filler $(C, D)$, total frontal reshaping with the biomaterial $(E)$, rotation of a wide galeal periosteal flap to cover the biomaterial with the use of fibrin glue (E,F). Total cover and protection of the bony substitute with a galeal-pericranial flap (G). Postoperative cap bandage for pressure dressing for a few days, helped the process of solidification and maintained the new forehead reshaping during the first 2-3 postoperative days $(G)$. Five years after surgery, a few small subcutanous irregularities became evident and were thus lipofilled $(\mathrm{H})$

Examination of the forehead and cranium revealed that the central frontal region was predominantly irregular with depressed areas and protuberances, which together, caused the grid effect. The patient appeared to be "frowning" as a result of the soft tissue of the 
forehead being entrapped into the fracture lines. Irregularity was confirmed on palpation, and depressed areas were particularly evident, indicating severe bony reabsorption. Plates and screws were evident and palpable subcutaneously. The patient complained of thermoceptive changes, abnormal changes in mimic movement of the frontal region, confirmed in the clinical examination, and marked aesthetic discomfort due to the severe cosmetic impact of the deformity.

Surgery was planned for removal of the plates and screws, remodeling of bony fragments and correction of forehead dysmorphology with CBVF, a biomaterial that was well suited to the characteristics of the case. CBVF is an injectable filler consisting of $40 \%$ hydroxyapatite, $60 \%$ calcium sulfate and antibiotic vancomycin hydrochloride(FIGURE1C,D). An advantage of this material is that it may be used as a reconstructive material in large body regions because it is easy to shape and also because initially it is a liquid and the setting time allows any necessary final retouching without lengthening operation time.
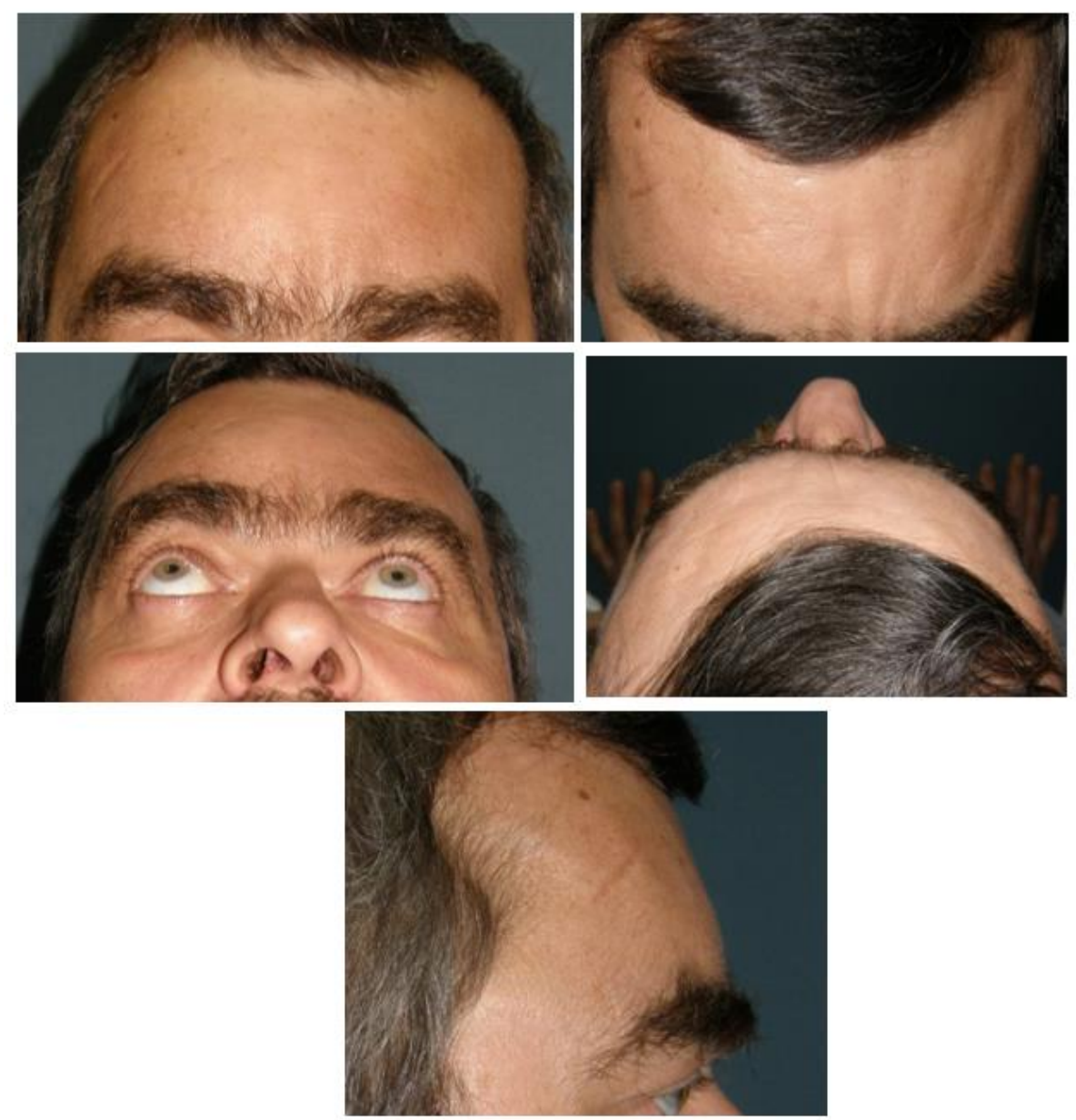

FIGURE 3 - A,B,C,D,E; Results, in different views, six years after forehead-supraorbital reconstruction with bone biomaterial $(A, B, C, D, E)$.

Other types of materials were not considered appropriate for this deformity because they were either too rigid or too liquid, making the reshaping more difficult, or were only available in formats that prevented acceptable application. The use of autologous bone was not taken into consideration; due to the size of the defect and because the patient had severe sequelae from previous severe craniofacial trauma.

Surgery was carried out under general anesthesia. Subperiosteal blunt dissection via 
the coronal approach was accomplished in the frontal region with exposure of the fronto-nasoorbital region. Due to scar tissue entrapped in the craniotomy lines, this phase caused problems. Severe fibrosis was also present and removed. The frontal region and the supraorbital ridge had severe deformities with marked irregularities .

All the hardware ,plates and screws, was completely removed. Some fragments were not integrated into the bone and were loose, most likely causing inadequate initial stabilization. The frontal region was totally reshaped using a burr to reduce the most evident irregularities and create absorptive surfaces for the biomaterial. The borders of the deformity were also remodeled by creating a borderline step to allow adequate retention of the material. CBVF was prepared and positioned to cover and fill the frontal area (FIGURE 2,A,B,C,D,E,F,G).

Relaxation incisions were carried out on the inner part of the coronal flap to allow more mobility of the flap itself. The biomaterial was then covered with rotation of a posterior wide galeal pericranial flap. A layer of fibrin glue was also positioned .

In the postoperative period a pressure dressing, with a cap bandage was positioned to maintain stabilization for about three-four days. This promoted stability and helped immediate postoperative solidification and shaping maintenance of the biomaterial, avoiding morphological alterations or movements of the newly reconstructed forehead-frontal bandeau. ${ }^{[10]}$ ).

A few years after surgery some small subcutaneous irregularities became evident; they were filled with a few $\mathrm{cc}$ of fat, lipofilling, improving the final forehead shape(FIGURE 2 H).

\section{RESULTS AND DISCUSSION}

No intraoperative or postoperative complications occurred, and the patient was discharged three days after surgery. Postoperative CT scan confirmed the new forehead shape and reduction of the supra-orbital deformities.

During a rather long follow up after surgery, stabilization of the result was maintained with satisfactory morphology and almost total disappearance of the fronto-orbital post-trauma bony and soft tissue deformities (FIGURE 3A,B,C,D,E).As an injectable synthetic bone void filler, CBVF has a unique ratio of hydroxyapatite and calcium sulfate designed to enable biomaterial to resorb at the same rate as that of bone formation.

Calcium sulfate acts as a resorbable carrier for hydroxyapatite which is highly osteoconductive and promotes bone ingrowth. With CBVF, de novo bone formation simultaneously occurs throughout the material and is fully remodeled into host bone within 6-12 months. Rapid bone remodeling reduces the risk of fracture, non union of fragments and possible re-infection. In conclusion, cranioplasty procedures and type of material to be used depend on the type of deformity, the surgeon's experience and background, and on the choice of material.[11] In this case the use of CBVF was easy to manage without any problems or morbidity. This material and the technique described above turned out to be a simple and effective method for restoring contour deformities and defects of the fronto-upper orbital area, and to obtain a long-term stabilized final result.

\section{DECLARATIONS}

Authors' Contributions: Luigi Clauser, Antonio Lucchi,Carolina Sannino designed the clinical data and research; Luigi Clauser performed surgical treatments; Luigi Clauser, Antonio Lucchi, Riccardo Luoni Orsi prepared the article.

\section{Availability of data and materials}

Not applicable

\section{Financial support and sponsorship}

None

\section{Conflicts of interest}

All Authors declared that there are no conflicts of interest

\section{Ethical approval and consent to participate}


Not applicable

\section{Consent for publication}

Written informed consent was obtained for all patient images.

\section{Copyright}

CThe Authors (2021)

\section{REFERENCES}

[1]. Green AK, Warren S, McCarthy JG. Onlay frontal cranioplasty using wire reinforced methyl methacrylate. J Craniomaxillofac Surg 2008:36:138Y142 doi: 10.1016/j.jcms.2007.05.008.

[2]. Lykins CL, Friedman CD, Costantino PD, et al. Hydroxyapatite cement in craniofacial skeletal reconstruction and its effects on the developing craniofacial skeleton. Arch Otolaryngol Head Neck Surg 1998;124:153Y159 doi: 10.1001/archotol.124.2.153.

[3]. David L, Argenta L, Fisher D. Hydroxyapatite cement in pediatric craniofacial reconstruction. J Craniofac Surg 2005;16:129Y133 doi: 10.1097/00001665-200501000-00026

[4]. Duman $H$, Deveci $M$, Uygur $F$, et al. Reconstruction of contour and anterior wall defects of frontal bone with porous polyethylene implant. J Craniomaxillofac Surg 1999;27:298Y301 DOI: 10.1054/jcms.1999.0902

[5]. Taghizadeh F, Kromer A, Laedrach K. Evaluation of hydroxyapatite cement for frontal sinus obliteration after mucocele resection. Arch Facial Plast Surg 2006;8:416Y422 . DOI: 10.1001/archfaci.8.6.416

[6]. Friedman CD, Costantino PD, Synderman $\mathrm{CH}$, et al. Reconstruction of the frontal sinus and frontofacial skeleton with hydroxyapatite cement. Arch Facial Plast Surg 2000;2:124Y129 DOI: 10.1001/archfaci.2.2.124

[7]. Hofmann et al. 'Autologous lliac Bone Graft Compared with Biphasic Hydroxyapatite and Calcium Sulfate Cement for the Treatment of Bone Defects in Tibial Plateau Fractures - A Prospective, Randomized, Open-Label, Multicenter Study.'J Bone Joint Surg Am. 2020;102:179-93. doi: 10.2106/JBJS.19.00680.

[8]. Nilsson, M. 'Injectable calcium sulphate and calcium phosphate bone substitutes'. Lund University. 2003. ISBN: 91-628-5603-0.

[9]. Kaczmarczyk et al. 'Complete twelve month bone remodeling with a bi-phasic injectable bone substitute in benign bone tumors: a prospective pilot study'. BMC Musculo. Dis. 2015;16:369. doi: 10.1186/s12891-015-0828-3
[10]. Clauser L. et al.Contouring of the forehead irregularities with Bone material.J Craniomaxillofac. Surg.2012;23:932-934 DOI $10.1097 /$ SCS.0b013e3182505593

[11]. Hettwer et al. 'Establishment and effects of allograft and synthetic bone graft substitute treatment of a critical size metaphyseal bone defect model in the sheep femur'. APMIS 2019; 127:53-63. doi: 10.1111/apm.12918 